\title{
Die handschriftlichen Vorarbeiten
}

\section{Editionsprinzipien}

Die Edition der handschriftlichen Vorarbeiten zum Quintus Fixlein folgt den im Band II/10.1 beschriebenen Prinzipien. ${ }^{32}$ Hier erfolgt eine kurze Zusammenfassung.

- Die einzelnen Handschriftenkonvolute werden in der von Goebel und Bernauer (vgl. Vorwort, S. VIII, Anm. 4 und Edition der handschriftlichen Vorarbeiten, 1. Beschreibung der Handschriften, S. XLIVff.) vorgenommenen Zählreihenfolge wiedergegeben. Sie beginnen jeweils auf einer neuen Seite. Unterabteilungen der Konvolute werden ebenfalls auf einer neuen Seite begonnen. Die von Völkel und Wernlein geschriebenen Passagen (Fasz. XIIIb, Konv. 25-30, vgl. Edition Vorarbeiten, S. 5,2-12,38, 21,2-22,20, 24,5-29,26) werden zusätzlich in kleinerer Schriftgröße gesetzt.

- Seitenwechsel werden durch senkrechte Striche $(\gg<)$ markiert. Die Blatt-, folio- bzw. die jeanpaulsche Zählung wird zusätzlich Höhe in der Marginalie angezeigt.

- Absätze werden immer dann verwendet, wenn das erste Wort der neuen Zeile unterstrichen ist und/oder wenn eine klare inhaltliche Abgrenzung vom vorausgehenden Absatz erkennbar ist. Ebenso wenn die vorausgehende Zeile nicht vollgeschrieben wurde bzw. der vorausgehende inhaltliche Kontext als abgeschlossen gelten kann.

- Der Abdruck des Texts erfolgt zeilengetreu. Über den Satzspiegel hinausgehende Zeilen werden umgebrochen und die Folgezeile durch eine Einrückung des Texts als solche markiert.

- Inhaltlich äquivalente Überschreibungen werden mit den üblichen Weichenzeichen für optionale Schreibweisen ( $<$ und $>-$ ) ediert. Einfügungen oberhalb der Zeile, die nicht der Kategorie >Weiche< zuzuordnen sind, werden in einer eigenen Zeile oberhalb der zugehörigen Zeile in korrektem Abstand zum Rand eingerückt abgedruckt.

- Unterstrichene einzelne Wörter oder Passagen werden im Sinne des Autors zur Gänze unterstrichen, d.h. es gibt keine partiell unterstrichenen Wörter, es sei denn, es ist klar festzustellen, dass Jean Paul einen bestimmten Wortbestandteil hervorheben wollte.

\footnotetext{
${ }^{32}$ Vgl. SW HKA II.10.1, Satiren und Ironien, hg. von BIRGIT SICK, S. XLVII-XCIII.
} 
- Jean Paul nutzte Schriftwechsel, um fremdsprachliche (meist lateinische) Ausdrücke zu kennzeichnen sowie um Textbereiche hervorzuheben. Die Edition bildet diesen Schriftwechsel durch einen Wechsel auf eine andere Type ebenfalls ab.

- Die vielfach vorkommenden Exzerpteverweise werden in der Form von Brüchen dargestellt, wobei der Zähler meist die Seite, seltener die Nummer, und der Nenner den Band des Exzerpthefts darstellt, wo das Notat zu finden ist. Wird der Nenner im Exzerpt durchgestrichen, so ist dies ein Verweis auf die mit IV nummerierten Hefte. Die Durchstreichung wird in der Edition mit zwei senkrechten Strichen neben dem Nenner symbolisiert.

- Unsichere Lesungen und Konjekturen werden unterpunktiert, nicht Entzifferbares mit $\times$ für Majuskel und $\times$ für Minuskel verzeichnet.

- Kursivierungen kennzeichnen Ergänzungen des vom Autor teilweise stark abgekürzten Textes durch die Herausgeber. Herausgebererläuterungen, die der Verdeutlichung des Textes dienen, sind ebenfalls kursiv gesetzt und stehen in eckigen Klammern.

- Emendationen erfolgen bei offenkundigen Schreibfehlern, Dittographien etc. Nicht wiedergegeben werden Verwendungsstreichungen und Federproben.

- Der Variantenapparat befindet sich auf derselben Seite wie der edierte Text, auf den er sich bezieht.

- Die originale Rechtschreibung Jean Pauls wird beibehalten (zu Jean Pauls eigenwilliger Orthographie vor 1804 - erst zu seinem 41. Geburtstag, am 21. März 1804, übernahm er das Regelwerk Adelungs - vgl. BIRGIT SICK SW HKA II 10.1, S. LIIff. und EDUARD BEREND SW HKA II.1, S. X und S. 351-357 sowie SW HKA II.2, S. 451-455 und SW HKA II.3, S. 371-375).

- Die Interpunktion folgt dem Handschriftenbefund.

- Die Edition bildet vom Autor gemachte Fußnoten als solche ab. Hochgestellte Zahlen mit vorausgehendem Leerzeichen sind Verweise auf Endnoten, die Jean Paul meist ans Ende der Konvolute stellte. Bei einigen Konvoluten (bspw. XIIIc/65) sind diese Endnoten nicht mehr vorhanden. 


\section{Siglen und Zeichen}

Textkonstitution:

Text:

$[$ Text $]$ :

$\times, \times$

Text

Text

$1 / 8$

$306 / 16$

$<,>-$

$2^{\mathrm{r}}$ etc.

1

Variantenapparat:

]

Text [...] Text ]

davor gestr.,

danach gestr.

(selten) darüber gestr. Streichungen Jean Pauls

aus

nachtr.

H

Sofortkorrektur

nachträgliche Einfügung
Autortext

Herausgebertext im edierten Text

nicht entzifferbarer Klein- bzw. Großbuchstabe

Unterstreichungen in der Handschrift

Lateinische Schrift in Handschrift

Exzerptverweise. Der Nenner bezeichnet den Band, der Zähler die Seite oder Eintragsnummer.

Weichenzeichen (öffnend und schließend);

bezeichnen zwei gleich gültige Formulierungen

Paginierungen am Außenrand der Seite

Anzeige des Seitenwechsels

Lemmazeichen

Kennzeichnung längerer Lemmata

bei einer Emendation wird mit $\mathrm{H}$ der Originalbefund angezeigt

Abkürzungen:

$\mathrm{H}$

Handschrift Jean Pauls

Bl.

Blatt

Bll.

Blätter

Fasz. $\quad$ Faszikel gemäß der Einteilung von Jean Pauls Handschriftlichem Nachlaß in der Staats-Bibliothek zu Berlin - Preußischer Kulturbesitz

Konv. Konvolut 
3. Verzeichnis der handschriftlichen Vorarbeiten und Drucke nach der Reihenfolge im Druck

\section{Billet an meine Freunde}

- Entwurf: HS, Fasz. XVII, Konv. 22, Nr. 15, Bll. 21 ${ }^{\mathrm{r}}-21^{\mathrm{v}}$ (undatiert) (vgl. Edition Vorarbeiten, S. 134,3-135,28)

- Billet an meine Freunde: Druck Quintus Fixlein, D1, 1796

- Billet an meine Freunde: Druck Quintus Fixlein, D2, 1801

\section{Mustheil für Mädgen}

\subsection{Der Tod eines Engels (vgl. SW HKA I.5, XIV)}

- Was der Tod ist: D 1 Deutsches Museum, Dezember 1788

- Was der Tod ist: D 2: SW HKA I.18 (1963), S. 95-98

- dazu: HS Fasz. XIIIc, Konv. 65, Tagebuchblätter, Nr.2, Bll. $3^{\mathrm{r}}-4^{\mathrm{v}}$ (27. Juni 1788) (vgl. Edition Vorarbeiten, S. 62,5-67,8)

- Der Tod eines Engels: nach Eduard Berend (SW HKA I.5, S. XIVf.) HS, Nachlaß Helene Köhler, datiert vom 28. Juni 1792, Germanisches Nationalmuseum Nürnberg, heute nicht mehr nachweisbar

- Der Tod eines Engels: Druck Quintus Fixlein, D1, 1796

- Der Tod eines Engels: Druck Quintus Fixlein, D2, 1801

\subsection{Der Mond. Phantasierende Geschichte}

Der Mond. Phantasierende Geschichte ist die zweimal und vollständig umgearbeitete Fassung des folgenden handschriftlichen Textes (vgl. SW HKA II.3, XXXf.):

- Das Leben nach dem Todte: HS Fasz. XIIIb, Konv. 34, Nr. 1, Bll. 1 ${ }^{\mathrm{r}}-3^{\mathrm{v}}$ (1790) (vgl. Edition Vorarbeiten, S. 44,2-48,21) Vgl. SW HKA II.3, S. 252-255

- Phantasien über den Mond: nach Eduard Berend (SW HKA I.5, S. XIVf.) HS, datiert 18. Juli 1792, im Nachlaß Helene Köhler, Germanisches Nationalmuseum Nürnberg; heute nicht mehr nachweisbar

- Aufzeichnungen und Einfälle zu Der Mond: HS, Fasz. XXVa, Konv. 2, Erstes Schmierheft (1794), Nr. 11, Bl. 10 $-10^{\mathrm{v}}$ (vgl. Edition Vorarbeiten, S. 151,10-152,29)

- Der Mond. Phantasierende Geschichte: Druck Quintus Fixlein, D1, 1796

- Der Mond. Phantasierende Geschichte: Druck Quintus Fixlein, D2, 1801 


\section{Einige Jus de Tablette für Mannspersonen}

\subsection{Ueber die natürliche Magie der Einbildungskraft:}

- Vorarbeiten, HS Faszikel XXVa, Konv. 3, Zweites Schmierheft 1795, Nr. 2,3, B1. 2r-2 (vgl. Edition Vorarbeiten, S. 154,3-156,9)

- Vgl. bereits Übungen im Denken. Erster Band. Dezember 1780. Bemerkungen, Nr. XXXII: SW HKA II.1, S. 74f. (Gedächtnis und Einbildungskraft scheint einerlei zu sein) Briefentwürfe vom 7. (an Matzdorff) und 9.02.1795 (an Emanuel)

- Brief vom 26.02.1795: Brief an Otto (SW HKA III.2, Seite 56) mit dem Manuskript zur Magie der Einbildungskraft; Ottos Antwort 26. 4. 1795 (SW HKA IV.2.1, 69ff.); Jean Pauls Umarbeitung mit Brief an Otto: 22. Mai 1795, SW HKA III.2, 86.

- Ueber die natürliche Magie der Einbildungskraft. Druck Quintus Fixlein, D1, 1796

- Ueber die natürliche Magie der Einbildungskraft. Druck Quintus Fixlein, D2, 1801

3.2 Des Amts-Vogts Josuah Freudel Klaglibel gegen seinen verfluchten Dämon

- Schilderung eines Zerstreueten: HS Faszikel XIIIb, Konv. 31, Satirische Übungen, Nr. 7, Bll. 35-40 (November 1790) (vgl. Edition Vorarbeiten, S. 37,5-43,12)

- Zum Text der frühen Fassung des Zerstreueten vgl. die fünfte der Satirischen Übungen vom November 1790 in SW HKA II.3, 312-317 (Schilderung eines Zerstreueten) und die Erläuterung ebd. XXXV. Ottos Kritik ist nach Berend (SW HKA I.5, XVII) nicht erhalten. Vgl. SW HKA III.1, 517.

- 1. Umarbeitung, 2. Fassung: Brief Jean Pauls vom 24.12.1790 an Otto (SW HKA III.1, 351ff.). Auch diese 2. Fassung ist nicht erhalten, wird von Berend aber aufgrund der Kritik Ottos rekonstruiert: Brief Ottos vom 3. und 4. Januar 1791: SW HKA IV.1, 215ff. und SW HKA IV.2, $621 \mathrm{f}$.

- 2. Umarbeitung, 3. Fassung: HS Fasz. XXVa, vgl. Ottos Brief vom 29. April 1795, SW HKA IV.2.1, S. 77f.

- Des Amts-Vogts Josuah Freudel Klaglibell gegen seinen verfluchten Dämon: Druck Quintus Fixlein, D1, 1796

- Des Amtsvogts Josuah Freudel Klaglibell gegen seinen verfluchten Dämon. Druck Quintus Fixlein, D2, 1801 
3.3 Es giebt weder eine eigennützige Liebe noch eine Selbstliebe, sondern nur eigennützige Handlungen

- Erste Niederschrift: HS Faszikel XIIIb, Konvolut 25-30, Es giebt keine eigennützige Liebe. Konvolut 1-5, 16 Bll., nach Kritik der Freunde Völkel und Wernlein im Sommer 1790 (vgl. Edition Vorarbeiten, S. 1,3-36,7)

- Zweifache Umarbeitung: Keine genaue Datierung, zwischen 1790 und 1795

- Letzte Redaktion: Schmierheft HS Fasz. XXVa, Konv. 3, Zweites Schmierheft, Nr. 13, 15, 16, Bll. $8^{\mathrm{r}}$ und $9^{\mathrm{r}}, 1795$ (vgl. Edition Vorarbeiten, S. $159,18-161,17)$

- Otto als Korrektor, vgl. Jean Pauls Brief vom 7. April 1795, SW HKA III.2, S. 74

- Es giebt weder eine eigennützige Liebe: Druck Quintus Fixlein, D1, 1796

- Es giebt weder eine eigennützige Liebe: Druck Quintus Fixlein, D2, 1801

3.4 Des Rektors Florian Fälbel's und seiner Primaner Reise nach dem Fichtelberg (Vgl. SW HKA I.5, XXf.)

- Des Subrektors Florian Fälbel's Reise mit seinen vielen Primanern: Ursprünglich könnte dies als Teil der 3. und 4. Szene des 2. Akts der Baierischen Kreuzerkomödie geplant gewesen sein. Notizen in HS Fasz. 17, Konv. 18, Einzelne Blätter aus verschiedenen Handschriften, Nr. 1-9, hier Nr. 2, $3\left(2^{\mathrm{r}}-3^{\mathrm{r}}\right)$ und 7-9 $\left(6^{\mathrm{r}}-9^{\mathrm{r}}\right)$, Anfang 1790 (vgl. Edition Vorarbeiten, S. 94,3-96,12 und 98,22-103,32)

- Florian Fälbel's Reise mit seinen Primanern: Erscheint als Titel in den geplanten Satiren vom 15. Juli 1790 (Vgl. Brief an Otto, 15. Juli 1790, SW HKA III.1, 300ff.)

- Manuskript, Erste Fassung: Erhalten haben sich ein Notizenblatt (02.02.1791; vgl. SW HKA III.1, 323f.) und einige ausgearbeitete Blätter. Vgl. Ottos briefliche Kritik: 5. bis 7. Februar 1791, SW HKA IV.1, 222ff.

- Manuskript geht an Otto (vgl. Jean Pauls Brief vom 18. März 1795, SW HKA III.2, S. 60)

- Des Rektors Florian Fälbel's und seiner Primaner Reise nach dem Fichtelberg: Druck Quintus Fixlein, D1, 1796

- Dass., D2, 1801

\section{Leben des Quintus Fixlein [Idylle]}

Meine Überzeugung, daß ich todt bin: D SW HKA II.3 S. 96-98 und S. 395-398, HS Faszikel XIIIc, Konvolut 29, Meine Überzeugung, dass ich tot bin, Bll. $1^{\mathrm{r}}-3^{\mathrm{r}}$ (1789) (vgl. Edition Vorarbeiten, S. 48,4-52,2)

Am 30. Januar 1795 geht der Text an Otto (SW HKA III.2, S. 48); dessen 
Kritik im Brief vom 7. bis 11. März 1795 (SW HKA IV.2.1, S. 41-48); am 31. März zum zweiten Mal an Otto (SW HKA III.2, S. 67); Ottos erneute Rezension: 3. bis 7. April 1795 (SW HKA IV.2.1, S. 50ff.)

Im Nachlaßverzeichnis Bde 1,2 (Ed. GoEBEL, BERNAUER) sind zur Idylle noch zahlreiche andere hs. Vorarbeiten verzeichnet:

- Fasz. XIIIc, Konv. 43, Vierzigtagsblatt, Nr. 1, Bll. 1-4 (1789) (u.a. 7. Zettelkasten) (vgl. Edition Vorarbeiten, S. 58,4-61,22)

- Fasz. XV, Konv.1, Quintus-Heft, Nr. 1-3 (1796-1800), Bll. 447-458 (vgl. Edition Vorarbeiten, S. 68-91,8)

- Fasz. XVII, Konv. 18, Einzelne Blätter aus verschiedenen Handschriften, Nr. 10-13, Bll. $10^{\mathrm{r}}-11^{\mathrm{v}}$ und $27^{\mathrm{r}}-30^{\mathrm{v}}, 27, \mathrm{~B} 11.126^{\mathrm{r}}-126^{\mathrm{v}}$ (u. a. 1. Zettelkasten, Postskript) (1794) (vgl. Edition Vorarbeiten, S. 104,1-118,25)

- Fasz. XVII, Konv. 22, Nr. 15, Bll. 21 $-21^{\mathrm{v}}$ (Billet an meine Freunde) (vgl. Edition Vorarbeiten, S. 134,4-135,28)

- Fasz. XXVa, Konv. 2, Erstes Schmierheft 1794, Nr. 1-13, Bll. 1 1 $-10^{\mathrm{v}}$ (1794) (vgl. Edition Vorarbeiten, S. 136,2-152,29)

- Fasz. XXVa, Konv. 3, Zweites Schmierheft 1795, Nr. 1-16, Bll. 1 $1^{\mathrm{r}}-7^{\mathrm{r}}$ (1795) (vgl. Edition Vorarbeiten, S. 154,3-160,18)

- Fasz. XXVa, Konv. 4, Drittes Schmierheft 1795, Nr. 2, Bll. 1 $-2^{\mathrm{r}}$ (Februar, März 1795) (vgl. Edition Vorarbeiten, S. 162,3-164,28)

- Fasz. XXVa, Konv. 7, Arbeitsheft Nr. 4, Nr. 11, Bll. 19, Bll. 34-35 und 38-39 (vgl. Edition Vorarbeiten, S. 165,4-170,2)

- Fasz. XXVa, Konv. 8, Mixta. Einzelne Blätter, Nr. 1,2, 8 (1796), Bll. $1^{\mathrm{r}}-1^{\mathrm{v}}$ (vgl. Edition Vorarbeiten, S. 171,3-173,3)

\section{Geschichte meiner Vorrede zur zweiten Auflage des Quintus Fixlein (vgl. SW HKA I.5, XXIVff.)}

- Fasz. XVII, Konv. 22, Nr. 15, Bll. 21 ${ }^{\mathrm{r}}-21^{\mathrm{v}}$ (vgl. Edition Vorarbeiten, S. 134,4-135,28)

- Fasz. XXVa, Konv. 7, Arbeitsheft Nr. 4 (1796), Nr. 11, 25, Bll. 19 und 38-39 (vgl. Edition Vorarbeiten, S. 167,5-168,2 und 168,6-170,2)

Erste Ankündigung des Textes an Verleger Lübeck: 5. August 1796, SW HKA III.2, 229

Am 22. August 1796 geht das Manuskript an Otto (SW HKA III.2, 237)

\subsection{Die Mondsfinsternis (Vgl. SW HKA I.5, XXV)}

Erster Entwurf: Neujahrswunsch für Renate Wirth, Brief Jean Pauls vom 3. Januar 1791, SW HKA III.1, 317ff.

Text im September 1796 an Charlotte von Kalb, 16. Oktober, SW HKA IV.2, $241 \mathrm{ff}$. 
Zweite Auflage, 1801 (vgl. SW HKA I.5,XXVf.)

Fasz. XXVa, Konv. 7, Arbeitsheft Nr. 4, Nr.23, Bll. 34-35 (1796) (vgl. Edition Vorarbeiten, S. 166,5-168,2) 